\title{
LORENTZ-VIOLATING PHOTONS WITH A MASS TERM
}

\author{
MAURO CAMBIASO \\ Departamento de Ciencias Físicas, Universidad Andrés Bello \\ Santiago, Chile \\ E-mail: mcambiaso@unab.cl \\ RALF LEHNERT \\ Indiana University Center for Spacetime Symmetries \\ Bloomington, IN 47405, USA \\ E-mail: ralehner@indiana.edu \\ ROBERTUS POTTING \\ CENTRA, Departamento de Física, Universidade do Algarve \\ 8005-139 Faro, Portugal \\ E-mail: rpotting@ualg.pt
}

\begin{abstract}
Perturbative calculations in quantum field theory often require the regularization of infrared divergences. In quantum electrodynamics, such a regularization can for example be accomplished by a photon mass introduced via the Stueckelberg method. The present work extends this method to the QED limit of the Lorentz- and CPT-violating Standard-Model Extension.
\end{abstract}

\section{Introduction}

Massive vector particles are of relevance for various subfields of physics including the weak interaction, test models for photon-mass searches, and the regularization of certain infrared divergences in quantum-field calculations. In this latter context, the mass term should be introduced such that the symmetries of the original model remain unspoiled. For a U(1) Lorentzinvariant gauge theory, this can be accomplished with the Stueckelberg method. ${ }^{1}$

In recent years, Lorentz-violating quantum field theories have become a focus of theoretical ${ }^{2}$ and experimental ${ }^{3}$ inquiry. Such theories also require the regularization of infrared divergences in some circumstances, and the question arises, as to whether the Stueckelberg method can be adapted to 
such Lorentz-breaking U(1) gauge theories. As in the Lorentz-invariant case, the adapted Stueckelberg method should be compatible with all symmetries of the theory to be regulated, so that the usual Stueckelberg approach can be employed for the Lorentz-violating extension of conventional QED. However, the Lorentz violation in this model allows a broader range of compatible Stueckelberg terms that may be introduced, and this freedom can then be used to streamline calculations in Lorentz-breaking QED.

The present work provides one possible class of extensions of the usual Stueckelberg procedure to Lorentz-violating QED. Section 2 gives a brief overview of the procedure at the lagrangian level. Some implications of the Lorentz-breaking Stueckelberg Lagrangian are discussed in Sec. 3.

\section{Lagrangian Analysis}

We begin with the usual free-photon Lagrangian in the minimal StandardModel Extension coupled to a conserved source $j^{\mu}$ :

$$
\mathcal{L}_{\gamma}=-\frac{1}{4} F^{2}-A \cdot j-\frac{1}{4}\left(k_{F}\right)^{\kappa \lambda \mu \nu} F_{\kappa \lambda} F_{\mu \nu}+\frac{1}{2} \epsilon^{\kappa \lambda \mu \nu}\left(k_{A F}\right)_{\kappa} A_{\lambda} F_{\mu \nu} .
$$

Here, Lorentz and CPT breakdown is controlled by the spacetime-constant backgrounds $k_{F}$ and $k_{A F}$.

The absence of Lorentz symmetry in the above Lagrangian (1) allows us to drop the requirement of Lorentz invariance for the mass-type term $\delta \mathcal{L}_{m}$ to be introduced for the photon. However, the inclusion of arbitrary Lorentz violation into $\delta \mathcal{L}_{m}$ may be problematic: consider the case in which $k_{F}$ and $k_{A F}$ are such that a subgroup of the Lorentz group remains unbroken. A regulator violating this residual symmetry may be undesirable, so that the breakdown of the remaining invariant subgroup may have to be excluded from $\delta \mathcal{L}_{m}$.

For our present purposes, however, we consider arbitrary Lorentz violation in $\delta \mathcal{L}_{m}$. This yields more general results also relevant for purposes other than infrared regularization. More specifically, we implement the Stueckelberg procedure by introducing a scalar field $\phi$ as follows: ${ }^{4}$

$$
\delta \mathcal{L}_{m}=\frac{1}{2}\left(\partial_{\mu} \phi-m A_{\mu}\right) \hat{\eta}^{\mu \nu}\left(\partial_{\nu} \phi-m A_{\nu}\right),
$$

where $m$ can later be identified with the photon mass and

$$
\hat{\eta}^{\mu \nu}=\eta^{\mu \nu}+\hat{G}^{\mu \nu}
$$

with $\hat{G}^{\mu \nu}$ a Lorentz-violating operator that may contain derivatives but is otherwise spacetime constant. The small number of Lorentz-symmetric pieces still contained in an arbitrary $\hat{G}^{\mu \nu}$ can be removed if necessary. 
As in the conventional Stueckelberg case, the key feature of $\mathcal{L}_{\gamma}+\delta \mathcal{L}_{m}$ is its invariance (up to total derivatives) under a local gauge transformation

$$
\delta A_{\mu}=\partial_{\mu} \epsilon(x), \quad \delta \phi=m \epsilon(x) .
$$

With the addition of $\xi$-type gauge fixing $\mathcal{L}_{\text {g.f. }}=-\frac{1}{2 \xi}\left(\partial_{\mu} \hat{\eta}^{\mu \nu} A_{\nu}+\xi m \phi\right)^{2}$ and a Faddeev-Popov contribution $\mathcal{L}_{\text {F.P. }}$, the model Lagragian $\mathcal{L}=\mathcal{L}_{\gamma}+\delta \mathcal{L}_{m}+$ $\mathcal{L}_{\text {g.f. }}+\mathcal{L}_{\text {F.P. }}$ becomes ${ }^{4}$

$$
\begin{aligned}
\mathcal{L}= & -\frac{1}{4} F^{2}-A \cdot j+\frac{1}{2} m^{2} A_{\mu} \hat{\eta}^{\mu \nu} A_{\nu}-\frac{1}{2 \xi}\left(\partial_{\mu} \hat{\eta}^{\mu \nu} A_{\nu}\right)^{2} \\
& -\frac{1}{2} \phi\left(\partial_{\mu} \hat{\eta}^{\mu \nu} \partial_{\nu}+\xi m^{2}\right) \phi-\bar{c}\left(\partial_{\mu} \hat{\eta}^{\mu \nu} \partial_{\nu}+\xi m^{2}\right) c \\
& -\frac{1}{4}\left(k_{F}\right)^{\kappa \lambda \mu \nu} F_{\kappa \lambda} F_{\mu \nu}+\frac{1}{2} \epsilon^{\kappa \lambda \mu \nu}\left(k_{A F}\right)_{\kappa} A_{\lambda} F_{\mu \nu} .
\end{aligned}
$$

Note that the scalar $\phi$ and the ghosts $c$ and $\bar{c}$ are now decoupled and can be integrated out. We can therefore disregard these fields in what follows.

\section{Equations of Motion}

The Lagrangian (5) yields the following equation of motion for the photon:

$$
\begin{aligned}
{\left[\eta^{\mu \alpha} \eta^{\nu \beta} \partial_{\mu}+\right.} & \left.\left(k_{A F}\right)_{\mu} \epsilon^{\mu \nu \alpha \beta}+\left(k_{F}\right)^{\mu \nu \alpha \beta} \partial_{\mu}\right] F_{\alpha \beta} \\
& +\left[m^{2} \hat{\eta}^{\mu \nu}+\frac{1}{\xi} \hat{\eta}^{\mu \alpha} \hat{\eta}^{\nu \beta} \partial_{\alpha} \partial_{\beta}\right] A_{\mu}=j^{\nu} .
\end{aligned}
$$

Taking the 4-divergence of this equation yields

$$
\left(\hat{\eta}^{\mu \nu} \partial_{\mu} \partial_{\nu}+\xi m^{2}\right)\left(\partial_{\alpha} \hat{\eta}^{\alpha \beta} A_{\beta}\right)=0 .
$$

Note that $\left(\partial_{\alpha} \hat{\eta}^{\alpha \beta} A_{\beta}\right)$ projects out one degree of freedom contained in $A_{\mu}$. Equation (7) establishes that this degree of freedom is not excited by the source $j_{\nu}$, so $\left(\partial_{\alpha} \hat{\eta}^{\alpha \beta} A_{\beta}\right)$ is an auxiliary mode. This is consistent with the expectation of three physical degrees of freedom for a massive vector field.

A plane-wave ansatz in Eq. (6) yields the model's dispersion relation; it has the following structure: ${ }^{4}$

$$
\frac{1}{\xi}\left(1+\frac{1}{4} \hat{G}_{\alpha}^{\alpha}\right)\left(\hat{\eta}^{\mu \nu} p_{\mu} p_{\nu}-\xi m^{2}\right) Q(p)=0 .
$$

The first factor does not contain physical modes. The $\left(\hat{\eta}^{\mu \nu} p_{\mu} p_{\nu}-\xi m^{2}\right)$ piece corresponds to the auxiliary mode governed by Eq. (7). The factor $Q(p)$ is associated with the physical degrees of freedom and has the structure

$$
Q=\left(p^{2}-m^{2}\right)^{3}+r_{2}\left(p^{2}-m^{2}\right)^{2}+r_{1}\left(p^{2}-m^{2}\right)+r_{0} .
$$

Here, the coefficients $r_{j}$ are coordinate scalars containing the Lorentzbreaking tensors $k_{F}$ and $k_{A F}$. They vanish in the limit $k_{F}, k_{A F} \rightarrow 0$. The 


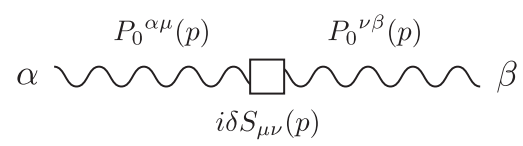

Fig. 1. Propagator insertion. The wavy lines denotes the conventional Lorentz-invariant Stueckelberg propagator $P_{0}$. The square box represents the leading-order Lorentzbreaking insertion given by Eq. (10).

explicit expressions for the $r_{j}$ can be found in the literature. ${ }^{4}$ Note that the physical dispersion relation (9) is consistent with the expectation of three conventional massive modes perturbed by small Lorentz violation.

The exact expression for the corresponding propagator is somewhat unwieldy. ${ }^{4}$ But for most applications only leading-order Lorentz-violating effects need to be taken into account. The Lorentz-breaking contributions can then be incorporated via this propagator insertion (see also Fig. 1): ${ }^{4}$

$$
\begin{aligned}
\delta S(p)^{\mu \nu}= & 2 i\left(k_{A F}\right)_{\alpha} \epsilon^{\alpha \beta \mu \nu} p_{\beta}-2\left(k_{F}\right)^{\alpha \mu \beta \nu} p_{\alpha} p_{\beta} \\
& +m^{2} \hat{G}^{\mu \nu}-\frac{1}{\xi}\left(\hat{G}_{\alpha}^{\mu} p^{\alpha} p^{\nu}+\hat{G}_{\alpha}^{\nu} p^{\alpha} p^{\mu}\right) .
\end{aligned}
$$

\section{Acknowledgments}

This work has been supported in part by the Indiana University Center for Spacetime Symmetries, by Universidad Andrés Bello under Grant No. UNAB DI-27-11/R, as well as by the Portuguese Fundação para a Ciência e a Tecnologia.

\section{References}

1. E.C.G. Stueckelberg, Helv. Phys. Acta 11, 225 (1938); Helv. Phys. Acta 11, 299 (1938).

2. See, e.g., D. Colladay and V.A. Kostelecký, Phys. Rev. D 58, 116002 (1998); V.A. Kostelecký, Phys. Rev. D 69, 105009 (2004); V.A. Kostelecký and R. Lehnert, Phys. Rev. D 63, 065008 (2001); A.J. Hariton and R. Lehnert, Phys. Lett. A 367, 11 (2007); R. Lehnert, Phys. Rev. D 74, 125001 (2006); M. Cambiaso et al., Phys. Rev. D 90, 065003 (2014).

3. See, e.g., V.A. Kostelecký and N. Russell, arXiv:0801.0287v7; M.A. Hohensee et al., Phys. Rev. Lett. 102, 170402 (2009); Phys. Rev. D 80, 036010 (2009); J.-P. Bocquet et al. [GRAAL Collaboration], Phys. Rev. Lett. 104, 241601 (2010); J.S. Díaz et al., Phys. Rev. D 88, 071902 (2013).

4. M. Cambiaso et al., Phys. Rev. D 85, 085023 (2012). 